\title{
LA CONSOLAZIONE DI DIO NELLE PROVE DEL MINISTERO EXEMPLA IN 2 COR. 1, 3-11
}

\author{
GOD’S CONSOLATION IN TESTS OF MINISTRY EXEMPLA IN 2 COR 1, 3-11
}

In the following article we have tried to analyze the consolation of God in the Pauline ministry tests. The reason that prompted me to address the topic of consolation of God, finds its explanation in the fact that too often we focus on the sufferings of this time without cast an eye over a horizon of hope. The works we have examined could be a source of consolation for the man who is living moments of trial. It is processed in the following way: First of all, it has made an etymological study of two terms: affliction and consolation, trying to see the various facets of Jewish antiquity, and in the texts of Sacred Scripture, even with short references to classical Greek. Later it saw the word in the Old and New Testament. Then, after a brief summary of the Second Epistle to the Corinthians, it analyzed the passage 2 Cor. 1.3 to 11 , which has been the subject of study and then move on to some issues, deepen through study of some authors who have addressed the issue. Particular attention was given to the sufferings of Christ that Paul experienced in his apostolate and their saving power for the believer.

Keywords: consolation, affliction, tribulation, ministry, tests

\section{Il concetto di afflizione}

Prima di affrontare il tema della consolazione di Dio è opportuno soffermarsi sul concetto di afflizione.

Il verbo greco $\theta \lambda i ́ \beta \varepsilon ı v$ che sta per «affliggere» che ha il significato di «schiacciare», insieme al sostantivo $\theta \lambda \tilde{i} \psi 1 \varsigma$, generalmente tradotto con i termini «afflizione», «oppressione», vengono usati nell'AT per indicare spesso la situazione di oppressione o di grave difficoltà sperimentata da Israele (cf. Es 3,9; 4,31; $1 \mathrm{Mac}$ 9,27). Il termine $\theta \lambda \tilde{i} \psi 1 \varsigma$, inoltre, è usato per indicare le tribolazioni del tempo finale in un contesto escatologico (cf. Dn 12,1; Ab 3,16; Sof 1,5), ma indica anche le sofferenze del giusto (Sal 33,20; 36,39; cf. 4Esdra 7,89; Apoc. Bar. 15,8; 48,50). ${ }^{1}$

\footnotetext{
${ }^{1}$ Cf. Schlier 1968.
} 
Nel NT il verbo $\theta \lambda i ́ \beta \varepsilon t v$ ricorre 10 volte, di cui 7 nel Corpus Paulinum $(3 \times$ in 2 Cor; $1 \times$ in $1 \mathrm{Ts}$; $2 \times$ in $2 \mathrm{Ts}$; $1 \times$ in Tm), mentre il sostantivo $\theta \lambda$ ĩ $\psi$ is è usato 45 volte, di cui ben 24 volte nel Corpus Paulinum ( $5 \times$ in Rm; $1 \times$ in 1Cor; $9 \times$ in 2 Cor; $1 \times$ Ef; $2 \times$ in Fil; $1 \times \mathrm{Col}$; $3 \times$ in $1 \mathrm{Ts} ; 2 \times$ in $2 \mathrm{Ts}$ ). Come nell'AT anche nel NT il termine può riferirsi alle sofferenze escatologiche, come la «grande tribolazione» di cui si parla in Mt 24,21 (cf. Mc 13,19), accompagnata da fenomeni cosmici (cf. Mc 13,24; Mt 24,29; Lc 21,25), ma anche a situazioni di persecuzione (cf. Mt 24,9; Mc 13,10; 1Ts 3,3; Ap 1,9).

Le tribolazioni che riguardano Paolo e i credenti sono messe in relazione con quelle di Cristo stesso (2Cor 4,10; Fil 3,10; 1Pt 413) e, generalmente, ciò che Paolo scrive circa le proprie sofferenze vale anche per tutti gli altri cristiani, sebbene l'Apostolo si ritenga colpito in maniera speciale dall'esperienza del dolore (2Cor 1,5). Essendo unite a quelle di Cristo, le tribolazioni dei cristiani, in un certo senso, sono necessarie (Gv 16,33; At 14,22). L'espressione più chiara la troviamo in 1 Ts 3,3, dove Paolo fa sapere di avere inviato Timoteo a raccomandare «affinché

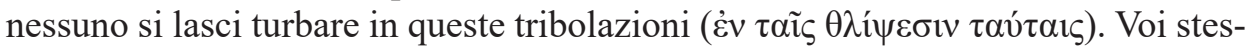
si, infatti, sapete che a questo siamo destinati». Paolo già le prevede per se stesso (1Ts 3,4; cf. At 20,23) e le sperimenta pesantemente durante il suo apostolato (2Cor 1,4.6.8; 2,4; 4,8; 6,4-5; 7,4-5; Ef 3,13; Fil 1,17; 4,14; 1Ts 3,4.7).

Ma le afflizioni non provengono solo dall'esterno, ma anche dall'interno della comunità cristiana (Fil 1,16-17: «gli uni annunciano il Cristo per amore ... gli altri per ambizione, senza sincerità, pensando di apportare afflizione alle mie catene»; cf. anche 2Cor 2,4).

Le afflizioni sperimentate personalmente da Paolo hanno anche finalità altruistica, come consolare gli altri $(2 \mathrm{Cor} 1,4)$ e ottenere consolazione e salvezza (2Cor $1,6)$, in quanto vengono sopportate come sofferenze di Cristo stesso (2Cor 1,5).

\section{Il concetto di consolazione}

Alle afflizioni subite da Israele e dai membri della comunità cristiana risponde Dio con la sua consolazione. Per comprendere pienamente il significato di tale iniziativa divina è utile soffermarsi brevemente sull'etimologia ebraica e greca dei termini che rendono l'idea di con-solazione o di conforto così come testimoniata nell'AT e nel NT.

\subsection{La consolazione nell'Antico Testamento}

In ebraico la radice $n h m$ sta alla base del binomio consolazione/consolare e originariamente significa «respirare profondamente», «gemere» e, nel senso causativo, «far respirare», «far tirare un fiato». Se l'etimologia ebraica sottolinea l'aspetto fisico-psicologico della consolazione (fare respirare, portare sollievo), la 
terminologia greca sottolinea piuttosto l'aspetto di incoraggiare, esortare, sostenere, confortare i sofferenti.

Composto dal verbo greco $x \alpha \lambda \varepsilon ́ \omega$ («chiamare»), il composto $\pi \alpha \rho \alpha x \alpha \lambda \varepsilon ́ \omega$ può assumere diverse accezioni: «pregare, esortare, ammonire, confortare». ${ }^{2}$ Insieme al termine corrispondente $\pi \alpha \rho \alpha ́ x \lambda \eta \sigma ı \varsigma$ il verbo $\pi \alpha \rho \alpha x \alpha \lambda \varepsilon \dot{\varepsilon} \omega$ è presente nella letteratura e filosofia greca, ma, per lo più, quando esso traduce il concetto di «conforto» si rimane nell'ambito di situazioni di lutto, come in una lettera a Falaride.

Nella versione dei LXX notiamo che la consolazione riguarda una persona addolorata per un lutto subito (Gen 24,67; 37,35; 38,12; 2Sam 12,24; Gb 29,25; Ger 16,$7 ; 38,15)$ ed è usata anche per esprimere le condoglianze (2Sam 10,2-3; $\mathrm{Gb} 42,11)$. Tuttavia, c'è da notare che l'accezione di consolazione divina o umana è praticamente assente nei libri dei LXX che non traducono, come i libri dei Maccabei, un originario testo ebraico.

Altri significati che questi determinati libri greci danno al termine $\pi \alpha \rho \alpha ́ \kappa \lambda \eta \sigma 1 \varsigma$ sono, per esempio, «preghiera» (1Mac 10,24), «assicurazione» (2Mac 7,24), mentre il verbo, invece, ha il senso di «incoraggiare» (1Mac 5,53; 12,50; 13,3; 2Mac 13,12). Una certa varietà di significati la troviamo in $2 \mathrm{Mac}$, dove $\pi \alpha \rho \alpha x \alpha \lambda \varepsilon \tilde{v} v$ ha il senso di «esortare», «incoraggiare» $(13,12)$, «sollecitare» $(4,34 ; 6,21)$, «rassicurare» $(13,23)$, «incitare» $(13,3 ; 14,25)$, «rafforzare» $(15,17)$, «proporre» $(11,15)$, «tranquillizzare» $(11,32)$, «invitare» $(12,3)$.

Stando ad un'antica interpretazione di Gen 5,29 il vino appare come il più antico dei consolatori della Bibbia e, in occasione delle visite di condoglianze, oltre alle parole di conforto, si offrivano anche il pane e la coppa di consolazione (Ger 16,5-7). Tuttavia, il consolare fa parte dell'opus proprium di Dio, sebbene l'officium consolandi, nell'AT, spettava a tutti e non solo, come nell'antichità classica, a determinate persone come ai familiari, agli amici o al filosofo consolatore. La Scrittura ci dà testimonianza dell'attività consolatrice di Dio nei confronti del singolo individuo (cf. Sal 22,4; 70,21; 85,17; 93,19), ma anche dell'intero popolo (cf. Is 51,12.19-23).

Nell'AT spesso si fa riferimento ad una falsa e vana consolazione, ad esempio quella che l'uomo cerca negli idoli o quella cercata nell'oro e nei beni materiali a cui si contrappone la vera consolazione, quella divina. Infine, bisogna notare che alcuni testi, come quello del Siracide, fanno riferimento perfino al concetto di autoconsolazione, in cui si raccomanda un lutto breve, in quanto il prolungarlo servirebbe solo a perpetuare il dolore (Sir 38,17-23; 30,21-25).

\subsection{La consolazione nel Nuovo Testamento}

Prendendo in esame l'uso di $\pi \alpha \rho \alpha x \alpha \lambda \varepsilon ́ \omega$ e $\pi \alpha \rho \alpha ́ x \lambda \eta \sigma ı \varsigma$ nel NT notiamo che il significato dei termini è strettamente collegato con l'evento salvifico, sebbene in

2 Cf. Helewa 1993. 
tutt'una serie di passi nel NT, come anche nell'AT, il verbo rimane perfettamente entro i limiti dell'uso linguistico comune.

Nella tradizione sinottica il verbo ha soprattutto il senso di «supplicare» o «implorare aiuto» e viene usato in modo particolare quando gli uomini si rivolgono a Gesù per essere guariti. Così, in Mt 8,5 il centurione supplica Gesù per la guarigione del servo paralizzato oppure gli anziani dei Giudei nello stesso episodio riportato da Lc 7,4. Ancora, nel vangelo di Marco troviamo il verbo nell'episodio della guarigione dell'indemoniato (Mc 5,18), dei numerosi malati (Mc 6,56//Mt 14,36), del lebbroso (Mc 1,40) e, per finire, nella commovente implorazione di Giairo per la guarigione di sua figlia (Mc 5,23//Lc 8,41).

Nel senso di «esortare» il verbo viene utilizzato soprattutto negli Atti e nelle lettere di Paolo e si riferisce a coloro che predicano la parola di salvezza. Il verbo $\pi \alpha \rho \alpha x \alpha \lambda \varepsilon \tilde{v} v$, quindi, viene a significare il messaggio salvifico che proviene dalla predicazione apostolica (cf. 2Cor 5,20: «noi fungiamo da ambasciatore per Cristo ed è come se Dio esortasse per mezzo nostro»).

Tra i diversi significati del verbo $\pi \alpha \rho \alpha \varkappa \alpha \lambda \varepsilon \varepsilon \tilde{v}$ nel libro degli Atti si possono riscontrare alcune sfumature come quelle di «invitare», «pregare di venire», «chiedere», «pregare» o anche «fare pressione». Similmente, in 2Cor 8,6; 9,5; 12,8 il verbo $\pi \alpha \rho \alpha x \alpha \lambda \varepsilon \tilde{i} v$ e il sostantivo $\pi \alpha \rho \alpha ́ x \lambda \eta \sigma ı \varsigma$ non hanno un significato religioso. I nostri termini, però, ricorrono nel contesto del discorso sulla colletta che, nella concezione paolina, è un vero atto culturale, mentre sono assenti nella lettera ai Galati, negli scritti giovannei e nella lettera di Giacomo.

Mail verbo ha anche il significato di «consolare» ed è usato, in modo particolare, nelle lettere paoline e nella lettera agli Ebrei per indicare la consolazione che viene dalla salvezza presente e futura di Dio.

Come si evince da Rm 15,4, Paolo, riferendosi all'AT, parla della consolazione che viene dalle parole scritte. Si tratta di quella consolazione utile a conservare la speranza e che trova la sua fonte ultima nel «Dio della pazienza e della consolazione» (Rm 15,5). In 2Ts 2,16-17 Paolo mette in evidenza il nesso della consolazione con l'atto di amore in Cristo Gesù e in Fm 7 l'aiuto che la comunità ha dato all'Apostolo è motivo di gioia e consolazione. ${ }^{3}$

La consolazione per Paolo è legata anche alla soddisfazione nel constatare nelle comunità la fede e l'affetto nei sui confronti (cf. 1Ts 3,7; 2Cor 7,6-7.13). Tuttavia, anche la consolazione che viene dagli uomini rimanda alla consolazione vera che viene da Dio stesso. Dio, infatti, viene lodato come «Dio di ogni consolazione»

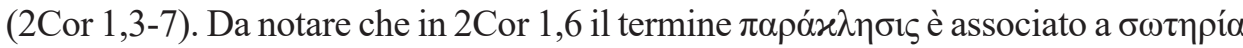
(«salvezza»): "Quando siamo tribolati, è per la vostra consolazione e salvezza». D'altra parte, la consolazione dei cristiani di Corinto consiste nella partecipazione alla consolazione provata da Paolo come conseguenza della loro partecipazione anche alle sue sofferenze. Da qui la ferma speranza che Paolo nutre per loro.

\footnotetext{
${ }^{3}$ Cf. Rosalba 2011.
} 
In 2Cor 1,5-7, come anche in 2Ts 2,16 e Rm 15,4, troviamo anche

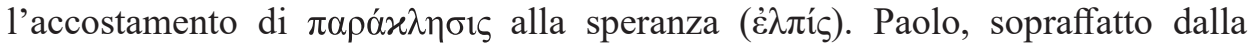
consolazione divina, consiglia ai Corinzi di «perdonare e consolare colui che lo aveva offeso» (2Cor 2,7). In tutti questi casi il rimedio contro le afflizioni consiste nella consolazione fatta con parole, ma la consolazione è dovuta anche ad eventi concreti, come l'arrivo di Tito (2Cor 7,6) o la liberazione di Paolo da un pericolo mortale (2Cor 1,3. 8-11) o anche la resurrezione di Eutico (At 20,12).

\section{Le afflizioni e la consolazione nella Seconda Lettera ai Corinzi}

La Seconda Lettera ai Corinzi, dove Paolo manifesta tutto se stesso, l'uomo, 1'Apostolo, il mistico, presenta molti punti oscuri che non conosciamo e che possiamo solo intuire. Nell'epistola, vibrano con energia i diversi sentimenti dell'Apostolo, la gioia il timore la speranza e la tenerezza. In essa Paolo esprime anche sentimenti di rammarico e di rimprovero di fronte a situazioni difficili che la chiesa di Corinto viveva. Inoltre, con la sua lettera Paolo ha la possibilità di giustificare la sua mancata visita, e mettere in guardia $i$ fedeli dai falsi profeti. Erano giunti, infatti, a Corinto dei nuovi evangelizzatori che non solo prendevano le distanze da Paolo, ma ne contestavano addirittura l'autorità. Essi sostenevano che l'Apostolo mercanteggiasse la Parola di Dio (2Cor 2, 14-17) e sfruttasse i Corinzi (cfr 2Cor 11, 7-15); inoltre faceva notare che il suo ministero non s'imponeva e non mostrava segni esterni potenti che ne convalidassero l'importanza, come confermerebbe un certo giudizio nei suoi confronti (2Cor 10, 10: «Le lettere -si dice- sono dure e forti, ma la sua presenza fisica è debole e la parola dimessa»). Questi missionari cristiani vantavano la loro origine e formazione ebraica, e affermavano che la legge data da Dio a Mosè non poteva essere trascurata perché su di essa si fondava l'alleanza. Si qualificavano, inoltre, come «apostoli», inviati di Cristo perché non solo erano in contatto con i suoi discepoli storici, ma potevano contare su esperienze particolari di rivelazione carismatica. In base a questi titoli di prestigio i nuovi missionari giudeo-cristiani non solo si facevano ospitare dai loro simpatizzanti a Corinto, ma pretendevano addirittura di essere ricompensati lautamente dalla comunità.

Informato di questa situazione, quindi, l'Apostolo tentò di intervenire personalmente facendo una rapida visita a Corinto subito dopo la partenza dei nuovi predicatori, ma si scontrò con i loro sostenitori e in modo particolare con chi li aveva accolti e ospitati. Paolo dunque, rientrato ad Efeso, scrisse una lettera ai Corinzi nella quale espresse tutta la sua delusione e amarezza per quello che gli era capitato nella loro città. Volendo rievocare quei momenti nell'attuale seconda lettera ai Corinzi, egli dice: «Vi ho scritto in un momento di grande afflizione e con il cuore angosciato, tra molte lacrime, però non per rattristarvi, ma per farvi 
conoscere l'affetto immenso che ho per voi» $(2$ Cor 2,4$) .{ }^{4}$ In questa lettera, scritta tra molte lacrime, Paolo invita i Corinzi a prendere dei provvedimenti nei confronti di chi, con il proprio atteggiamento, non solo lo ha offeso personalmente, ma ha screditato anche tutta la comunità cristiana.

Per accertarsi che la lettera abbia sortito l'esito desiderato e completare l'opera di riconciliazione con i Corinzi Paolo invia in Acaia il compagno Tito. Nel frattempo l'Apostolo, costretto a lasciare Efeso, si sposta verso Tróade e poi raggiunge la Macedonia, dove finalmente incontrerà Tito di ritorno da Corinto. Paolo è consolato dalle buone notizie portate da Tito, come testimonia il passo di : 2 Cor 7,6-7 «[...] Dio, che consola gli afflitti, ci ha consolati con la venuta di Tito; non solo con la sua venuta, ma con la consolazione che ha ricevuto da voi. Egli ci ha annunciato il vostro desiderio, il vostro dolore, il vostro affetto per me, cosicché la mia gioia si è ancora accresciuta.». Tuttavia le polemiche a Corinto non erano ancora spente e Paolo non indugia nello scrivere una missiva con l'intento di stroncare, una volta per tutte, le polemiche di quei calunniosi pseudo-apostoli.

Dopo aver guardato alla Seconda Lettera ai Corinzi nel suo insieme, focalizziamo ora la nostra attenzione sulla pericope 2 Cor 1,3-11 proponendone, anzitutto, un commento esegetico e scendendo poi, nello specifico della tematica.

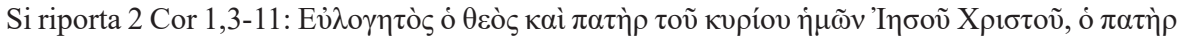

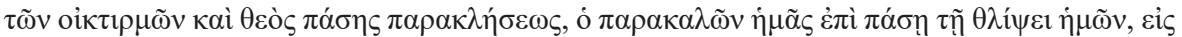

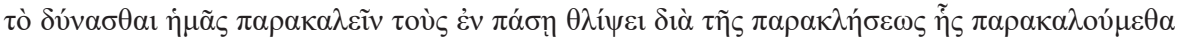

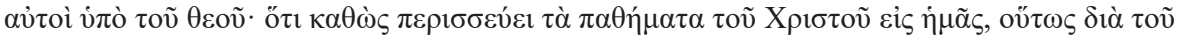

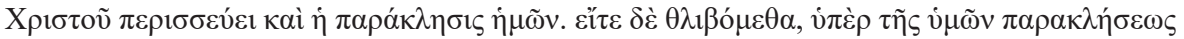

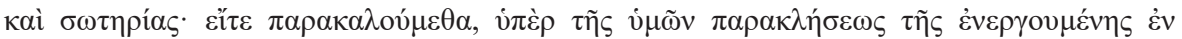

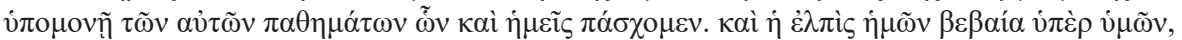

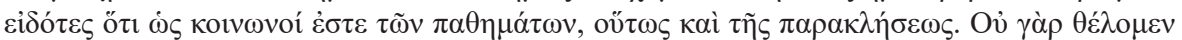

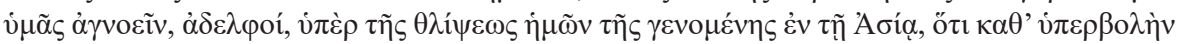

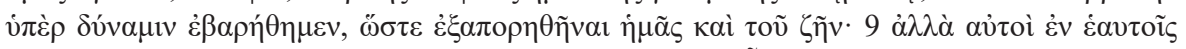

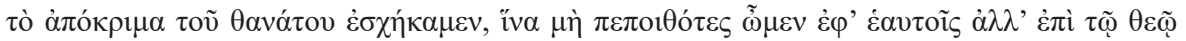

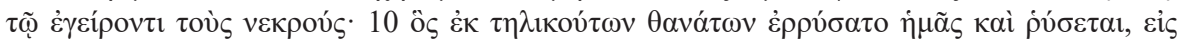

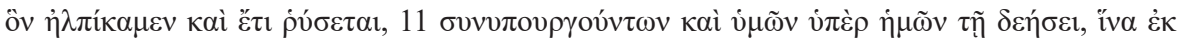

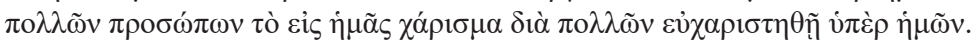

Sia lodato il Dio e Padre del Signore nostro Gesù Cristo, Padre delle misericordie e Dio di ogni consolazione, che ci consola in ogni nostra afflizione, per poter noi consolare coloro che sono in ogni afflizione mediante la consolazione con la quale noi stessi siamo consolati da Dio. Perché come le sofferenze di Cristo abbondano in noi così, per mezzo di Cristo, abbonda anche la nostra consolazione. Se siamo afflitti è per la vostra consolazione e salvezza, se siamo consolati è per la vostra consolazione che opera nella sopportazione delle stesse sofferenze che anche noi soffriamo. E la nostra speranza nei vostri riguardi è salda, convinti che come voi siete partecipi delle sofferenze così [lo siete] anche della consolazione. Infatti, non vogliamo che ignoriate, o fratelli, la nostra tribolazione che ebbe luogo in Asia, che fummo oppressi oltremisura, al di là delle forze, tanto che disperammo, anche della vita. Ma proprio noi

\footnotetext{
${ }^{4}$ Cf. Fabris 2009.
} 
abbiamo avuto in noi stessi la condanna di morte, affinché non confidassimo su noi stessi, ma in Dio che risuscita i morti, il quale ci ha liberato e ci libererà da un così grande pericolo di morte. In lui abbiamo riposto la speranza che ancora [ci] libererà, mentre anche voi cooperate con la preghiera per noi, affinché il beneficio (dato) a noi da molte persone sia per molti motivo di rendimento di grazie per noi.

\subsection{La struttura della pericope}

In conformità al formulario epistolare di Paolo, il prescritto $(1,1-2)$ precisa i mittenti e i destinatari della missiva, i cristiani di Corinto e dell'Acaia, ai quali Paolo e il suo discepolo Timoteo, come mittenti, augurano la grazia e la pace di Dio. Subito dopo il prescritto ai vv.3-7 non troviamo il ringraziamento (cfr. Rm 1,8-15; 1 Cor 1,4-9; Fil 1,3-1; 1 Ts 1,2-10), ma la benedizione..$^{5}$ Alcuni studiosi propongono di limitare l'unità ai vv.3-7, poiché al v.8 si trova un'espressione che solitamente ci si

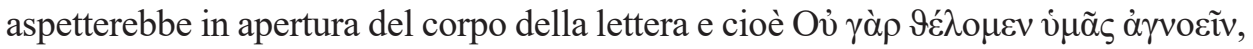
$\grave{\alpha} \delta \varepsilon \lambda$ poí, (non vogliamo infatti che voi ignoriate fratelli), con un cambiamento stilistico che parrebbe una cesura tra $\mathrm{i}$ vv. 3-7 e 8-11, una formula adoperata quattro volte nelle lettere paoline dopo il ringraziamento ( $\mathrm{Rm} \mathrm{1,13;} 2 \mathrm{Cr}$ 1,8; Fil 1,12; Col $2,1)$, quando è già entrato o sta per entrare nel corpo della lettera. Ma a suffragare 1'unità dei vv. 3-11, ci sono elementi stilistici e tematici. Infatti nei vv. 9-10 notiamo la presenza del motivo escatologico, che anticipano 4,7-14, e di quello della salvezza dai pericoli, tipici del genere benedizione e di ringraziamento; quest'ultimo presente anche al v. 11. In 1,8-11 è evidente inoltre il climax dell'esordio, con la figura dell' accumulatio nella descrizione della prova subita in Asia e della salvezza (i tempi verbali: presente, aoristo e futuro; il ringraziamento da parte di «molti», che fa da inclusione con l'eulogia in 1,3a). Bisogna notare, poi, che a partire da 1,12 i temi e il vocabolario cambiano.

Quindi, il nostro brano appartiene al genere letterario dell'eulogia, ovvero della benedizione, ed è usato in contesti liturgici per benedire Dio. Posto all'inizio della lettera, esso serve ad introdurre i temi da trattare e, cioè l'A postolo al servizio della consolazione di Dio, la sofferenza come strumento principale di questo ministero. Questa benedizione esercita anche una sorta di captatio benevolentiae, riconoscibile nella cura di Paolo di esprimere l'affetto per i Corinzi (la reciprocità di 1,6-7 e il tono confidenziale di 1,8-11) allo scopo di accrescere la fiducia vicendevole.

Dopo 1'indirizzo della lettera, il brano si articola nel seguente modo: ${ }^{6}$

- vv. 3-4: benedizione e motivo dell' eulogia

- vv. 5 -7: le spiegazioni

${ }^{5}$ Cf. Fil 1,3-11; 1 Ts1,2-10. Nel NT si trovano altre benedizioni introduttive solo in Ef 1,3-14 e 1 Pt 1, 3-12.

${ }^{6}$ Cf. Giacomo 2007. 
- vv. 8-10: il concreto motivo della lode

- vv. 11: il ringraziamento da parte di molti

Sono assenti le preghiere dell' Apostolo per i suoi destinatari, anche se vi è un accenno alle preghiere che i Corinzi hanno rivolto a Dio a favore dell'Apostolo (v. 11). Manca la lode per la virtù dei destinatari, evidentemente perché Paolo non vuole elogiare coloro che hanno messo in discussione il suo apostolato e, ancor più, il suo Vangelo. «Soggetto principale è Dio (vv. 4-10), mentre Paolo e i collaboratori sono soggetti in frasi subordinate (vv. 4c-6c) oppure in proposizioni principali, con verbi al passivo (vv. 6ab. 8b-d) o dal significato ricettivo («ab-biamo ricevuto» : v. 9a).» ${ }^{7}$ Paolo svolge solo l'azione di benedire, (v.3), l'avere ferma speranza (v. 7), la decisione di rendere partecipe i Corinzi (v. 8a), il confidare in Dio (v. 9b), dunque l'enfasi è posta sull'agire di Dio. «La crudezza della prova è descritta con un crescendo di intensità; altrettanto vale per il motivo della salvezza da parte di colui che risuscita i morti, visto nella sua dimensione escatologica» ${ }^{8}$ L'idea dell'abbondanza del potere salvifico di Dio, è messa in evidenza dalla struttura dinamica dei vv. 8-11. Paolo confida nel «Dio che risuscita i morti», nella forza di colui che ha risuscitato Gesù Cristo e, siccome egli vede la propria vita come una situazione di morte, vede la salvezza dalla tribolazione come risurrezione.

\subsection{Commento esegetico}

v.3: Sia lodato il Dio e Padre del Signore nostro Gesù Cristo, Padre delle misericordie e Dio di ogni consolazione.

La formula della lode (vv. 3-4) è di uso liturgico ebraico, ma qui viene cristianizzata dall'aggiunta «Padre del Signore nostro Gesù Cristo». In questa eulogia Dio è benedetto soprattutto perché «Padre della Misericordia», espressione tipica della preghiera sinagogale (cf. la preghiera delle Diciotto benedizioni: «Ascolta la nostra voce, Signore Dio nostro, padre misericordioso!»), L'espressione, però, nel nostro caso non si limita all'attribuzione della bontà a Dio Padre, ma evoca il suo carattere di misericordia offerto all'umanità, secondo quanto si legge in Es 34,6 e Sal 25,6; 68,17: «Volgiti a me nella tua grande tenerezza». Il Dio della misericordia, si esprime nelle consolazioni e, attraverso $\pi \alpha ́ \sigma \eta \zeta$, («di ogni»), si indicano le infinite sfumature degli interventi di grazia confortatrice di Dio in favore dei suoi figli, come è capitato anche a Paolo.

v. 4: che ci consola in ogni nostra afflizione, per poter noi consolare coloro che sono in ogni afflizione mediante la consolazione con la quale noi stessi siamo consolati da Dio.

\footnotetext{
Cf. Ibidem.

${ }^{8}$ Cf. Ibidem.
} 
Il versetto 4 ci offre il motivo della lode a Dio, cioè l'aver sperimentato la Sua conso-lazione. L'azione confortatrice di Dio non è venuta mai meno nella vita di Paolo ed è stata fondamentale non solo affinché l'Apostolo fosse consolato, ma anche perché dalla consolazione di Dio egli riceveva la forza per andare avanti nel suo ministero nonostante i pericoli, e, molto di più, per renderlo capace di confortare, a sua volta, coloro che si trovavano in situazioni analoghe. Ciò avveniva non per motivi di affinità psicologica, ma per Grazia, attraverso l'azione confortatrice del Padre. In questo versetto trovano spazio due diverse tipologie di consolazione: da un lato abbiamo, infatti, la consolazione che giunge all'Apostolo da parte di Dio, dall'altro la consolazione degli afflitti attraverso la mediazione di Paolo. ${ }^{9}$

v.5: Perché come le sofferenze di Cristo abbondano in noi così, per mezzo di Cristo, abbonda anche la nostra consolazione.

In questo versetto, viene fatto riferimento, invece, alle sofferenze di Cristo e dei Corinzi. Non si tratta soltanto di dolori o di prove simili a quelli sofferte da Cristo o subiti per amore di lui, ma è Cristo stesso, nell'esperienza mistica di Paolo, che opera, soffre e vince nei suoi fedeli, grazie alla comunione che hanno instaurato con lui nel Battesimo (cfr Rm 6,8; Fil 3,11).

v.6: Se siamo afflitti è per la vostra consolazione e salvezza, se siamo consolati è per la vostra consolazione che opera nella sopportazione delle stesse sofferenze che anche noi soffriamo.

La vita dell'Apostolo è radicalmente orientata verso i suoi figli spirituali. Egli la vede inclusa e rapportata in un disegno divino. Tribolazione e consolazione dell'Apostolo sono in vista della «salvezza» dei Corinzi. È lecito chiedersi a cosa si riferisce Paolo quando parla di salvezza. Si potrebbe, anzitutto, riferire alla liberazione dalle tristezze e dalle tribolazioni che si erano addensate sulla comunità di Corinto. Il conforto divino che defluisce da Dio in Paolo e da questi nei Corinzi già si manifesta nel fatto che essi possono sopportare con forza le medesime sofferenze dell'Apostolo; parole queste che sembrano esprimere più un augurio che una realtà, come risulta essere messo in evidenza nel versetto seguente. ${ }^{10}$

v.7: E la nostra speranza nei vostri riguardi è salda, convinti che come voi siete partecipi delle sofferenze così [lo siete] anche della consolazione.

Nel v. 7 Paolo afferma di avere una speranza salda nei confronti dei Corinzi, perché così come sono partecipi delle sofferenze di Cristo lo saranno anche della consolazione. Non si parla di una partecipazione futura ma attuale. La Thrall

\footnotetext{
${ }^{9}$ Cf. Lorusso 2007.

${ }^{10}$ Cf. Rossano 2008.
} 
ritiene che «la comunione alle stesse sofferenze» sia data dalle comuni sofferenze causate dall'opposizione incontrata sia da Paolo che dai Corinzi. ${ }^{11}$

v.8: Infatti, non vogliamo che ignoriate, o fratelli, la nostra tribolazione che ebbe luogo in Asia, che fummo oppressi oltremisura, al di là delle forze, tanto che disperammo, anche della vita

I motivi per cui Paolo ha elevato la benedizione a Dio vengono elencati nei versetti 8-11 e riguardano eventi passati, dai quali l'Apostolo è stato liberato. La rievocazione avviene con verbi al passato, aoristo e perfetto. Dalle parole del testo, sembrerebbe che il rischio corso in Asia sia stato tremendo, tanto da essere stata in pericolo la sua stessa vita. Nel testo l'Apostolo non fornisce alcun particolare sulla vicenda. Questo silenzio da parte di Paolo può trovare giustificazione o nel fatto che ai Corinzi era sufficiente anche un rapido cenno alla questione essendo a loro gli eventi abbastanza noti o forse Paolo si è limitato ad una generica allusione perché il suo fine non era cronistico. Alcuni commentatori ipotizzano che il fatto, di cui Paolo parla, sia avvenuto ad Efeso. ${ }^{12}$ Questa supposizione non convince però tutti, poiché anche in altre circostanze la città viene menzionata (cfr. 1 Cor $15,32 ; 16,8)$. Probabilmente qui mancano dettagli poiché l'Apostolo voleva, in realtà, attirare l'attenzione del lettore solo su due aspetti per lui importanti: l'aspetto pedagogico della terribile esperienza e l'intervento liberatore di Dio. Egli parla al plurale includendo, così, anche il compagno Timoteo; tuttavia non è chiaro se il pericolo sia stato corso da entrambi. Resta oscura anche la causa di questo pericolo. Varie le ipotesi proposte per l'identificazione del fatto oggettivo: R. Yates ${ }^{13}$ pensa alla drammatica giornata dell'insurrezione di Efeso (At 19,21-41), nella quale Paolo ebbe salva la vita come per miracolo. Altri sostengono che si tratti del combattimento contro le belve avvenuto nella stessa città e riferito da Paolo in 1 Cor 15,32, oppure di un processo subito dall'Apostolo con il conseguente imprigionamento accaduto sempre ad Efeso, sulla base di 2 Cor $11,23 .{ }^{14} \mathrm{G}$. H. Rendall ipotizza si tratti del dispiacere provato da Paolo a causa della ribellione degli stessi Corinzi. ${ }^{15}$ Mentre E. B. Allo pensa, invece, ad una grave malattia che poteva condurlo alla morte. ${ }^{16}$

Ad ogni modo Paolo fa di quella prova, qualunque essa sia stata, un'occasione per rafforzare i suoi rapporti con la comunità di Corinto, che in quel momento, nonostante le continue tribolazioni che aveva dovuto affrontare, appariva ben predisposta alla sua parola.

v.9: Ma proprio noi abbiamo avuto in noi stessi la condanna di morte, affinché non confidassimo su noi stessi, ma in Dio che risuscita i morti

${ }^{11}$ Cf. Thrall 2007.

${ }^{12}$ Cf. Ibidem.

${ }^{13}$ Cf. Yates 1981.

${ }^{14}$ Cf. Thrall 2007.

${ }^{15}$ Cf. Rendall 1909.

16 Cf. Allo 1956. 
La prima parte del versetto sembrerebbe accentuare quanto già detto nel v. 8, parlando di sentenza di morte ( non fornisce ulteriori infor-mazioni dal punto di vista materiale sulla tipologia della tribolazione, ma descrive l'atteggiamento interiore dell'Apostolo in quella situazione di pericolo mortale. L'introduttiva congiunzione avversativa $\dot{\alpha} \lambda \lambda \dot{\alpha}$. introduce il motivo di tale dolorosa esperienza: una pedagogia divina (ìv $\alpha \mu \grave{\eta}$ $\pi \varepsilon \pi$ oł ${ }^{\prime} \tau \varepsilon \zeta \dot{\omega} \mu \varepsilon v$, «perché non confidassimo») finalizzata alla scoperta della fede nel Dio che risuscita i morti; motivo questo, presente nella preghiera delle diciotto benedizioni ( $\ll \mathrm{Tu}$ sei potente in eterno, Signore: tu riscatti i morti e sei grande nel salvare!», lo stesso motivo lo troviamo anche in $\mathrm{Rm} \mathrm{4,17:} \mathrm{"che} \mathrm{dà}$ vita ai morti e chiama all'esistenza le cose che ancora non esistono»). Tutto è stato provvidenziale nel dramma vissuto da Paolo ed egli è stato condotto dalla forza di un'esperienza limite a spostare decisamente ogni sua fidu-cia da se stesso in Dio.

v.10: il quale ci ha liberato e ci libererà da un così grande pericolo di morte. In lui abbiamo riposto la speranza che ancora $[\mathrm{ci}]$ libererà

L'Apostolo comprende la propria situazione come stato di morte e la liberazione come salvezza dal giogo della morte e, poiché ha sperimentato sia la morte che il potere di vita di Dio ovvero il potere escatologico del Cristo risorto, ora proclama apertamente la propria fiducia nel futuro intervento salvifico di Dio.

v. 11: mentre anche voi cooperate con la preghiera per noi, affinché il beneficio (dato) a noi da molte persone sia per molti motivo di rendimento di grazie per noi.

L'Apostolo ricorda ai suoi interlocutori, di pregare per lui. La liberazione divina nelle future afflizioni, infatti, è un dono che i Corinzi possono invocare da Dio mediante la preghiera. Si deve rendere grazie a Dio perché salvando l'Apostolo da un pericolo mortale, ha reso possibile e continuerà a rendere possibile l'opera di Paolo. Nel culto deve salire a Dio un ringraziamento collettivo per la salvazione dell'Apostolo che qui è detta «dono di grazia» $(\chi \alpha ́ \rho ı \mu \mu \alpha)$. Il ringraziamento del v.11 e la lode del v. 3 fanno sì che la pericope abbia un'unità linguistica e teologica in sé conchiusa. ${ }^{17}$

\subsection{Le sofferenze di Cristo e di Paolo}

Abbiamo potuto notare, analizzando la pericope oggetto di studio, come rivesta particolare importanza il tema della sofferenza di Cristo e di Paolo, che, d'altra parte, è presente in tutto l'epistolario paolino.

\footnotetext{
${ }^{17}$ Cf. Lang 2004.
} 
Già in 1 Ts 1,6, l'Apostolo parla implicitamente di sé e di coloro che ha convertito come imitatori del Signore per via della loro sopportazione nelle afflizioni. Egli può anche aver considerato la sofferenza che il suo ministero apostolico comportava come un mezzo per diventare imitatore di Cristo e, così, essere più conforme a Lui. Per via del Battesimo tra l'Apostolo e Cristo si è instaurata una comunione mistica, per mezzo della quale le sofferenze vissute dallo stesso Paolo non sono una mera imitazione delle sofferenze di Cristo, ma una ve-ra e propria partecipazione alle stesse. In questo senso scrive la Thrall: «E’ vero che Paolo non mette esplicitamente in relazione il Battesimo con la sofferenza, ma l'idea della morte con Cristo secondo Rm 6,3, conseguenza e significato del battesimo, è connessa in Fil 3,10 alla sofferenza. In conformità ininterrotta alla morte di Cristo, Paolo parteciperà alle Sue sofferenze (egli mira a esprimere $\tau \grave{\eta} v$

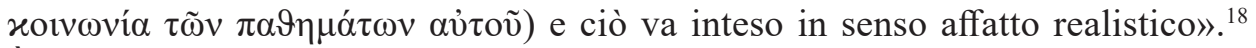
È importante, comunque, sottolineare che le sofferenze dell'Apostolo si possono leggere in chiave cristologica in riferimento al Signore risorto e glorificato dal quale egli non si separa. Per Paolo sopportare le tribolazioni è il più grande segno di apostolato, perché l'Apostolo, così facendo, si identifica con il Signore crocifisso. ${ }^{19}$ E' inevitabile, per chi sta annunciando Cristo, essere esposto ad ostilità e sofferenze, che sono una componente essenziale della vita cristiana

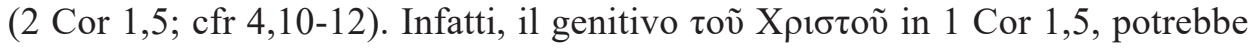
non essere un genitivus subiectivus, cioè un genitivo che fa da soggetto, ma

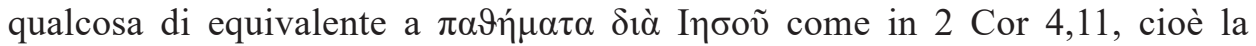

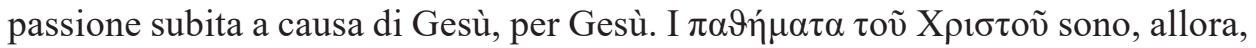
le sofferenze di Paolo e dei Corinzi (cfr. anche 4,10 e Fil 3,10). La loro natura di «passione di Cristo» deriva dal fatto che sia lui che i destinatari le sopportano a causa di Gesù che si rende presente con il suo potere salvifico nella vita dei singoli e nella vita dell'intera comunità. Altre proposte di interpretazione di questa sofferenza non si adattano al contesto apostolico nella lettera. Inoltre, la partecipazione alle sofferenze non può consistere in una comunione soltanto mistica, in quanto le tribolazioni possono essere di diverso genere. Ciò che conta è che sempre si tratta della sofferenza di Cristo, sia in Paolo che nei Corinzi. Le prove e le difficoltà del ministero di Paolo e degli apostoli sono interpretate, dunque, come una partecipazione alle sofferenze di Cristo. Il collegamento tra le sofferenze di Cristo e quelle di Paolo può essere delucidato attraverso l'idea di Chiesa come Corpo di Cristo. Secondo Paolo l'unione tra Cristo e le membra del suo corpo comporta che le sofferenze dei cristiani siano non solo una continuazione di quelle di Cristo, ma anche un loro completamento $(\mathrm{Col}$ 1, 24). Questo non significa che la passione di Cristo sia manchevole e siano necessarie le sofferenze dell'A postolo per completarla.

\footnotetext{
18 Thrall 2007.

19 Cf. Lorusso 2001.
} 
Le traduzioni a riguardo di Col 1,24, infatti, sono di solito inesatte. L'ha fatto osservare, a ragione, l'ultimo commento pubblicato sulla lettera ai Colossesi, di J.N. Aletti. ${ }^{20}$ Per migliorare lo stile della frase di Paolo i traduttori, infatti, modificano un po' l'ordine delle parole. Sembra poca cosa, ma, in realtà, cambia il senso.

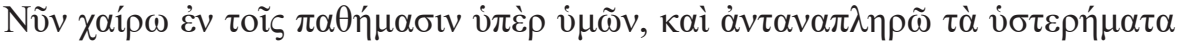

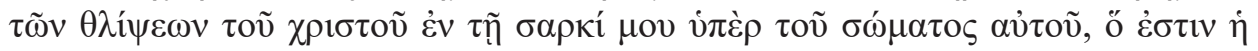

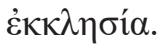

Il testo è per lo più così tradotto: «Sono lieto delle sofferenze che sopporto per voi e completo nella mia carne quello che manca ai patimenti di Cristo, a vantaggio del suo corpo che è la Chiesa».

Questa traduzione sembra far dire a Paolo che la passione di Cristo è stata manchevole di qualcosa, e Paolo avrebbe l'ambizione di completare lui stesso ciò

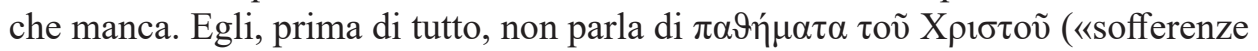
di Cristo») ma di $\theta \lambda i \tilde{u} 1 \varsigma$ («tribola-zione»), infatti l'espressione «nella mia carne» non si trova prima, ma dopo le parole «che manca alle tribolazioni di Cristo». La frase, quindi si dovrebbe tradurre: «Completo quello che manca nella mia carne alle tribolazioni di Cristo», oppure: «quello che manca alle tribolazioni di Cristo nella mia carne». Sulla stessa scia si colloca Manicardi, ${ }^{21}$ secondo il quale, stando al testo greco, la frase va tradotta in questo modo : «Io trovo la mia gioia nelle mie sofferenze per voi e completo ciò che manca alle tribolazioni di Cristo nella mia carne, per il suo corpo, che è la chiesa». Quindi, non è alla passione di Cristo che manca qualcosa, ma è alla partecipazione dei credenti alle sofferenze di Cristo che manca e mancherà sempre qualcosa.

Ogni vocazione cristiana comprende, quindi, una parte di tribolazioni che deve essere attuata. In questo senso, Paolo dice che egli completa ciò che manca all'applicazione della passione di Cristo nella sua esistenza, mostra la positività di questa passione, al punto da dire: «sono lieto delle sofferenze che sopporto per voi». Egli è infatti, convinto della fecondità di questa partecipazione alla passione di Cristo e vede che la partecipazione alla passione è condizione per partecipare alla risurrezione.

\subsection{I cristiani consolati da Dio mediante Paolo}

Il tema della consolazione non è presente solo nella lettera ai Corinzi, ma occupa grande spazio in tutti gli scritti paolini. Essi, indirizzati alle chiese, sono destinati ad essere letti e meditati di credenti lungo il loro cammino di fede, soprattutto nei momenti di afflizione di prova.

\footnotetext{
${ }^{20}$ Cf. Aletti 2011.

${ }^{21}$ Cf. Manicari 2006.
} 
Da una lettura attenta di 2 Cor 1,6 si ricava un rapporto stretto tra le afflizioni dell'Apostolo e la consolazione che da esse ne scaturiscono, consolazioni che non giovano solo all'Apostolo ma, attraverso di lui, giungono all'intera comunità di Corinto, una $\pi \alpha \rho \alpha ́ \kappa \lambda \eta \sigma ı \varsigma$ operata da Dio e ministrata da Paolo a beneficio dei credenti afflitti. È facile intuire l'importanza che acquista questo concetto nello sforzo operato da Paolo nel suo ministero per far comprendere e insegnare ai suoi ascoltatori il significato del soffrire terreno nella luce di Cristo e nel mistero dei segni divini. ${ }^{22}$ La consolazione ha una matrice teologica. All'origine, infatti, vi è la misericordia di Dio, di cui soggetto secondario e strumento per l'esortazione è il ministro; in questo modo la consolazione di Dio si riversa anche sui cristiani di Corinto mediante Paolo e i suoi discepoli, i quali, essendo stati confortati da Dio, diventano capaci di consolare a loro volta gli altri cristiani sofferenti. L'Apostolo è così convinto che i Corinzi, rimanendo uniti a lui e agli altri ministri del Vangelo, potranno perseverare nella fede pur affrontando le stesse tribolazioni. ${ }^{23}$ Tuttavia, secondo la Thrall, sarebbe un errore insistere troppo sul ruolo di intermediario svolto dall'Apostolo, in quanto si andrebbe a escludere la possibilità che Dio possa consolare direttamente i Corinzi. Infatti, se si prendono in considerazione le due ricorrenze di $\pi \varepsilon \rho \iota \sigma \sigma \varepsilon v ́ \omega$ al versetto v. 6 possiamo notare come, essendo stato segnato da un'intensa afflizione, l'Apostolo, riceve un'inconsueta e intensa consolazione divina, la quale diventerà, a sua volta, dimostrazione della potenza consolatrice di Dio. ${ }^{24}$ Già al v.4 Paolo aveva affermato che l'afflizione sperimentata è un presupposto per ricevere la consolazione, della quale diventerà tramite per gli altri. In seguito, al v. 6, l'Apostolo approfondisce il concetto dicendo che la sua afflizione è finalizzata non solo alla consolazione dei lettori, ma anche alla loro salvezza. La salvezza di cui parla Paolo, ha un aspetto escatologico, è cioè finalizzata alla restituzione della dignità della gloria divina perduta nell'uomo. Il processo mediante il quale ciò dovrà compiersi è già innescato per mezzo dell'annuncio del Vangelo ( $\mathrm{Rm} 1,16)$ e, proprio per questo, chi lo annuncia è soggetto ad afflizioni di ogni genere (2 Cor 4,7-12). L'annuncio salvifico giunge agli ascoltatori proprio nel contesto delle afflizioni; per questo Paolo può affermare che il suo soffrire è per amore di coloro che sta evangelizzando.

Inoltre si constata una stretta connessione tra le sofferenze di Paolo e quelle dei suoi lettori. L'Apostolo, infatti, afferma che le sofferenze dei suoi lettori sono le sue stesse sofferenze (v.6). Ad ogni modo, in queste sofferenze, effettive o solo prospettate ai Corinzi, si esercita la consolazione di Dio mediante l'Apostolo, di conseguenza i Corinzi sono resi capaci di sopportare la sofferenza, attraverso, l'incoraggiamento fiducioso di Paolo e attraverso la dimostrazione che nella sua stessa persona si rende evidente la potenza salvifica di Dio (v.7). ${ }^{25}$

22 Cf. Helewa 1993.

${ }^{23}$ Cf. Manzi 2002.

${ }^{24}$ Cf. Thrall 2007.

${ }^{25}$ Cf. Ibidem. 
Da questa esperienza Paolo ricava il principio generale secondo il quale Dio ci fortifica nelle nostre afflizioni. Inoltre, coloro che sono resi forti hanno la possibilità di rafforzare gli altri sia sostenendoli e incoraggiandoli, sia mostrando loro comprensione e compassione. Da ciò si ricava che la Grazia opera attraverso strumenti umani con la stessa persona di Paolo. I Corinzi vedono operante la Grazia di Dio nella consolazione e nel superamento delle prove sofferte da Paolo; essi sanno, quindi, di poter a loro volta sopravvivere nelle prove allo stesso modo in cui è accaduto a Paolo. ${ }^{26}$ In 2 Cor 1, 5-7 si chiarisce il modo in cui Paolo è reso confortatore della sua comunità, indicando non solo che l'Apostolo soffre allo stesso modo di Cristo e che le sue sofferenze sono sopportate proprio a causa di Lui, ma se ne ricava addirittura una concezione mistica, in quanto Cristo rivive nell'esperienza di Paolo e dei credenti il suo mistero pasquale. ${ }^{27}$ Paolo comprende che la sua vera esistenza si è trasformata in una permanente manifestazione della vicenda umana di Cristo, in particolare della sua passione e risurrezione, riconoscendo, in questo modo, che la sua vita è un tutt'uno col ministero apostolico. In quanto unito a Cristo, egli ne riceve la consolazione, ma non è il solo, infatti essendo unita alle sofferenze di Cristo, anche la comunità di Corinto riceverà la stessa consolazione. L'Apostolo e la sua chiesa, quindi, camminano insieme mossi da un conforto che si concretizza nella costanza, nel coraggio, nella perseveranza nelle mille difficoltà e avversità. E' possibile comprendere in quest'ottica come l'esperienza paradossale della tribolazione e della speranza, della sofferenza e della fiducia in Dio, che emerge dai ricordi personali narrati in 2 Cor 1,8-11, caratterizza il ministero apostolico di Paolo (cfr. 6,8-10. 7,4). In questa fiducia in Dio ogni Apostolo riflette il mistero pasquale di Cristo nelle difficoltà che il ministero comporta. Oltretutto, Paolo vive le prove del ministero in una prospettiva ecclesiale. In particolare, in 2 Cor 1,3-11, Paolo fa seguire alla benedizione elevata a Dio il ricordo della sua esperienza di sofferenza, dalla quale ha appreso, che non si deve confidare se non in Dio (v.9). Egli interviene nella vita dei cristiani per consolarli nelle loro afflizioni (cfr. vv. 3-4). La prova che l'Apostolo sostiene gli consente di dare testimonianza e di partecipare ai suoi fratelli nella fede, all'evento della salvezza che lo ha pienamente coinvolto. Lorusso afferma che questo annuncio, in modo analogico diventa "vangelo" (cfr.

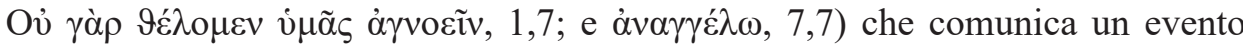
salvifico o un'esperienza di salvezza. Esaminando l'argomentazione, si può notare la funzione rivelatoria del discorso paolino e la sua portata apostolica in quanto comunicazione dell'opera di Dio. Ma oltre che testimone, Paolo è "strumento" della Pasqua di Cristo nel senso che in lui e tramite il suo soffrire, si diffonde nei destinatari l'efficacia del ministero pasquale e quindi della consolazione. ${ }^{28}$

\footnotetext{
${ }^{26}$ Cf. Murphy-O' Connor 1993.

${ }^{27}$ Cf. Barbaglio 1980.

${ }^{28}$ Cf. Lorusso 2001.
} 
E' importante notare che, questa consolazione non coincide con una passiva rassegnazione di fronte alle tribolazioni, ma, nella capacità di affrontarle con gli stessi atteggiamenti di Cristo (cfr. Fil 2,5), ovvero con la solidarietà di chi consola coloro che soffrono (cf. 2 Cor 1,4). ${ }^{29}$

\section{Conclusioni}

Al termine di questo nostro studio sul tema della consolazione presente nella seconda lettera ai Corinzi possiamo trarre alcune conclusioni.

Notiamo anzitutto che la consolazione non è un tema presente solo nella pericope che è stata presa in esame, ma anche in tutto il corpo paolino.

Paolo fa della consolazione una pietra miliare del suo ministero apostolico. Egli, infatti, vive il suo apostolato non solo portando il primo annuncio del Vangelo, ma sente forte il bisogno di continuare ad accompagnare il cammino di fede di quanti, attraverso di lui, sono diventati credenti. La sua passione apostolica si carica ancora di più nel momento in cui $\mathrm{i}$ credenti vivono situazioni di forte afflizione e dolore. Proprio in questi casi, come più volte abbiamo potuto evidenziare nel lavoro, Paolo si sente vicino a coloro che sono suoi fratelli nella fede e la sua parola evangelizzatrice giunge a loro nella duplice funzione di esortare chi soffre a restare saldo nella fede che ha abbracciato e, contemporaneamente, di consolare e dare speranza. È, quindi, questo il punto centrale della $\pi \alpha \rho \alpha ́ x \lambda \lambda \eta \sigma ı \varsigma$ di Paolo: esortare e consolare.

Il nostro lavoro ci ha permesso di comprendere, inoltre, il motivo per cui l'Apostolo può far giungere questa parola di consolazione agli afflitti. Egli ha avuto la possibilità di vivere in prima persona l'esperienza della consolazione nelle afflizioni, nelle prove e nelle numerose sofferenze subite a causa del Vangelo. Forte della sua esperienza personale, infatti, Paolo si fa ministro di consolazione. Egli si sente, allora, uno strumento nella mani di Dio, il quale può servirsi dell'Apostolo per far giungere conforto e consolazione ai sui figli che soffrono.

Da qui emerge una qualità particolare della consolazione che, evidentemente, non rap-presenta semplicemente un alleviare la pena di qualcuno mediante delle parole confortanti, ma richiama la potenza salvifica di Dio. È questo l'aspetto più interessante che si è cercato di mettere in risalto. Per Paolo la sofferenza non è un ostacolo, ma piuttosto un corroborante dell'apostolato, come i cataloghi peristatici (2Cor 4,8-9; 2Cor 6,4-5.8-10; 2Cor 11,23-33) mettono bene in evidenza, portando addirittura l'Apostolo a vantarsi di questi aspetti cosiddetti di debolezza.

In modo particolare, il ministero apostolico della consolazione trova le sue radici nel mistero pasquale di Cristo. Paolo comprende bene che le sue sofferenze, come quelle dei cristiani di Corinto, possono ricevere il dono della grazia divina

\footnotetext{
${ }^{29}$ Manzi 2002.
} 
e della consolazione in quanto unite al mistero pasquale. L'Apostolo unito alla passione e risurrezione di Cristo, sperimenta il dono della consolazione divina nei momenti della prova e del dolore; questa esperienza gli permette di diventare strumento nelle mani di Dio, il quale fa giungere ai sofferenti la propria consolazione. Inoltre, esortando, e confortando i cristiani afflitti, l'Apostolo memore della sua esperienza, li invita a diventare anch'essi ministri di consolazione.

Come lo vive Paolo il credente e come lo esercita Paolo il ministro, questo della $\pi \alpha \rho \alpha ́ x \lambda \eta \eta \sigma \varsigma$ esortatrice-consolatrice risulta essere una di quelle attività che meglio evidenziano la ricchezza e la grandezza dell'esistenza cristiana. È un'affermazione lucida della potenza del Vangelo come anche dell'immanenza viva della Grazia di Cristo, una celebrazione possiamo dire del Dio fedele che conferma misericordiosamente nei suoi figli l'opera del suo amore.

\section{Bibliografia}

Aletti, J. N. (2011). Lettera ai Colossesi. Introduzione, versione, commento. Bologna: EDB.

Allo, E.B. (1956). Saint Paul Seconde Épître aux Corinthiens. Paris: Etudes Bibliques.

Barbaglio, G. (1980). Le lettere di Paolo, 1. Roma: Borla.

Bieder, W. (2004). 9גĩ廿ıs. In H. Balz, G. Schneider (eds.). Dizionario esegetico del Nuovo Testamento. Brescia: Paideia, (= seconda edizione tedesca 1992), 1644-1649.

Fabris, R. (2003). Lettere di Paolo. Vicenza: ISG edizioni.

Fabris, R. (2009). Paolo l'apostolo delle genti. Milano: Paoline.

Girlanda, A. (2006). Nuovo Testamento. Balsamo: San Paolo Cinisello.

Helewa, G. (1993). «Un ministero paolino: consolare gli afflitti». Teresianum 44 (1993). 3.

Lang, F. (2004). Lettere ai Corinti. Brescia: Paideia.

Lorusso, G. (2001). Il ministero Pasquale di Paolo in 2 Cor 1-7, le implicazioni del soffrire e gioire per il Vangelo. Roma: Vivere in.

Lorusso, G. (2007). La seconda Lettera ai Corinzi introduzione versione e commento. Bologna: EDB.

Manes, R. (2011). Lettera a Tito e a Filemone. San Paolo: Cinisello Balsamo.

Manicardi, L. (2006). L'umano soffrire. Qiqajon: Comunità di Bose.

Manzi, F. (2002a). «Il Padre della consolazione (2 Cor 1,1-11)». Parole di Vita 5 (2002), 10-20.

Manzi, F. (2002b). Seconda lettera ai Corinzi, nuova versione introduzione e commento. Milano: Paoline.

Marconcini, B. (2007). Profeti e apocalittici. Torino: Elle di ci.

Murphy O'Connor, J. (1993). La teologia della Seconda Lettera ai Corinti. Brescia: Paideia.

Murphy O'Connor, J. (2007). Paolo, Un uomo inquieto, un apostolo insuperabile. San Paolo: Cinisello Balsamo.

Murphy O’Connor, J., Vita di Paolo. (1997). Brescia: Paideia.

Novum Testamentum Graece, (2012). E. Nestle et K. Aland (eds.). Stuttgart: Deutsche Bibelgesellschaft. Pitta, A. (2006). La seconda lettera ai Corinzi. Roma: Borla.

Rendall, G.H. (1909). The Epistles of St. Paul to the Corinthians. London: Macmilian.

Rossano, P. (2008). Lettere di S. Paolo. S. Paolo: Cinisello Balsamo.

Schlier, E. (1968). 9גĩuıs. In Grande Lessico del Nuovo Testamento IV. Brescia: Paideia.

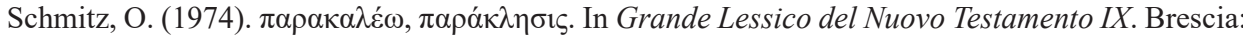
Paideia.

Thrall, M.E. (2007). 2 Corinti, 1. Brescia: Paideia. 